\title{
Oncological and functional results of extraperitoneal laparoscopic radical prostatectomy
}

\author{
TAO ZHENG, XU ZHANG, XIN MA, HONG-ZHAO LI, JIANG-PIN GAO, WEI CAI, \\ GUANG-FU CHEN, JUN DONG, BAO-JUN WANG and TAO-PING SHI \\ Department of Urology, Chinese People's Liberation Army General Hospital, \\ Military Postgraduate Medical College, Haidian, Beijing 100853, P.R. China
}

Received January 23, 2012; Accepted May 9, 2012

DOI: $10.3892 / \mathrm{ol} .2012 .737$

\begin{abstract}
The oncological and functional results of 329 cases in a population treated with extraperitoneal laparoscopic radical prostatectomy (ELRP) were evaluated retrospectively. A total of 329 inconsecutive patients with prostate cancer (PCa) who underwent ELRP were retrospectively analyzed. The median initial prostate-specific antigen (PSA) level was $17.35 \mathrm{ng} / \mathrm{ml}$. The median biopsy Gleason score was 7.77. Patients with a T2 or T3a clinical stage had received preoperative neoadjuvant hormonal therapy (NHT) for 3 to 9 months prior to ELRP. No conversion or re-intervention were observed. The median time for anastomosis, surgery time and postoperative catheterization time were $13.0 \mathrm{~min}, 90.0 \mathrm{~min}$ and 6 days, respectively. The median estimated blood loss was $75 \mathrm{ml}$. There were 12 temporary urinary leakages requiring prolonged catheterization to 14 days. There was 1 case of deep vein thrombosis, 1 case of alimentary tract hemorrhage and 7 cases of anterior urethral stricture. The median follow-up time was 27 months. A total of 17 patients were lost during the follow-up period. No rectal injury, lymphocele, incision hernia, postoperative persistent urinary leak or anastomotic stricture occurred. Younger patients ( $\leq 67$ years of age) had a more rapid recovery of continence and a better postoperative potency. The overall positive surgical margin rate was $16.7 \%$, which correlated with the pathological stage and Gleason score, respectively (both $\mathrm{P}<0.001)$. A total of $89(28.6 \%)$ patients were diagnosed with biochemical recurrence. The initial PSA value, PSM, pathological stage and Gleason score were identified as independent prognostic factors for biochemical recurrence-free survival using multivariate analysis. Our results demonstrated that preoperative NHT had significant effects on the pathological Gleason score $(\mathrm{P}<0.001)$ and surgical margin $(\mathrm{P}=0.027)$, but no
\end{abstract}

Correspondence to: Dr Xu Zhang, Department of Urology, Chinese People's Liberation Army General Hospital, Military Postgraduate Medical College, 28 Fuxing Road, Haidian, Beijing 100853, P.R. China

E-mail: xuzhangcn@yeah.net

Key words: prostate cancer, laparoscopic radical prostatectomy, follow-up, continence, potency, biochemical recurrence significant impact on biochemical recurrence $(P=0.202)$. The reproducibility of ELRP has been proven as a reliable curative treatment in Western countries during the last 15 years. Due to the increase in PCa patients, the results of our study may aid surgeons who use ELRP for the first time.

\section{Introduction}

Prostate cancer ( $\mathrm{PCa}$ ) is the second most frequently diagnosed type of cancer, and in 2008, it was the sixth leading cause of cancer mortality in males worldwide (1). Although the incidence of $\mathrm{PCa}$ in China is not as high as that in Europe and North America, it is currently on the increase. Gu $(2,3)$ evaluated the incidence of $\mathrm{PCa}$ at 187 hospitals based in 26 Chinese provinces. The overall incidence rate of $\mathrm{PCa}$ in 1997 was $1.5 \%$ (1389/95749). Between 1951 and 1960 the incidence rate of PCa at the Institute of Urology, Beijing University, Beijing, China, was $0.6 \%$. However, between 1991 and 1997 this rate has increased to $3.4 \%$ in total.

As a curative treatment for localized $\mathrm{PCa}$, laparoscopic radical prostatectomy has been performed in Western countries for almost 15 years. However, it remains a great challenge to many Chinese urologists. A narrower pelvis of Asian males compared with Caucasian males increases the difficulty of performing laparoscopic radical prostatectomy. A smaller incidence of PCa in China compared to Western countries means that surgeons require a longer time to become experienced. To the best of our knowledge, published studies decribing laparoscopic radical prostatectomy exceeding 300 Chinese cases are rare.

Between March 2005 and March 2010, we performed 329 cases of extraperitoneal laparoscopic radical prostatectomy (ELRP) with running urethrovesical anastomosis using the single needle method (4). In this study, we evaluated the oncological and clinical results after ELRP.

\section{Patients and methods}

Patient characteristics. All 329 patients had been confirmed as localized $\mathrm{PCa}$ by examinations using magnetic resonance images and transrectal ultrasound guided biopsies, with negative radionuclide bone scans (PSA $\geq 20 \mathrm{ng} / \mathrm{ml}$ and Gleason scores $\geq 7$ ). The median age and median initial prostate-specific 
antigen (PSA) levels are shown in Table I. The percentage of initial PSA, clinical stage and biopsy Gleason scores are shown in Table II.

A total of 77 patients with T2 or T3a PCa clinical stage had received neoadjuvant hormonal therapy (NHT) for 3-9 months prior to ELRP, while 94 patients had received NHT for $<3$ months. NHT comprised combined androgen blockade with luteinizing hormone-releasing hormone analogue (goserelin acetate) injections monthly and antiandrogen (bicalutamide, $50 \mathrm{mg}$ ) daily. A total of 30 patients had previously been treated with transurethral prostate resection (TURP) and ELRP was performed in these patients after an interval of 3 months.

Patients with localized PCa of clinical stage T1b-2c (NOM0) with a life expectancy of $>10$ years were candidates for ELRP. In each case, a bilateral pelvic lymph node dissection (PLND) was performed according to these indications: enlarged lymph nodes identified in magnetic resonance images, PSA $\geq 20 \mathrm{ng} / \mathrm{ml}$, Gleason scores $\geq 7$ or G3 tumor. Candidates for bilateral neurovascular bundles (NVB) sparing procedures were patients with normal preoperative potency, $\mathrm{T} 1$ or $\mathrm{T} 2$ diseases, $\mathrm{PSA}<10 \mathrm{ng} / \mathrm{ml}$ and Gleason scores $\leq 7$.

All patients consented to the use of their clinical data for this study. The Human Ethics Review Committee of Chinese People's Liberation Army General Hospital approved the study protocol.

Surgical technique. ELRP was performed using the technique as described by Stolzenburg et al (5), but with certain modifications. While Stolzenburg et al introduced the method of 5 -trocars placement, the number and position of the trocar placement was different in our study, in which 4 trocars were applied to complete the surgery (Fig. 1). Stolzenburg et al used an interrupted suture for urethrovesical anastomosis, whereas we preferred a running urethrovesical anastomosis using a $25 \mathrm{~cm}$ 2-0 Monocryl suture with a UR-6 tape needle. The initial suture was performed outside-in through the bladder neck at the 3 o'clock position and a knot was tied with a $3-\mathrm{cm}$ end outside on the posterior lip of the bladder neck. Another suture at the nearby position was performed outside-in through the bladder neck and inside-out through the urethra at the corresponding position. Under the guidance of a Foley catheter, clockwise running sutures were performed from 3 o'clock to 9 o'clock every 1 o'clock with slight traction. After gentle traction was applied to each suture to bring the bladder neck in contact with the urethra without excessive tension, the locking suture was performed at 9 o'clock. The running sutures were continued from 10 o'clock to 2 o'clock every 2 o'clock. The needle went inside-out of the urethra near the 3 o'clock suture and a knot was tied at the tail end after completing the full circumference. The surgeries were performed by the same experienced surgeon (XZ).

Postoperative treatment and follow-up. The patients were treated with antibiotics to prevent infection until removal of the stitches approximately 7 days postoperatively. Patients received patient-controlled intravenous analgesia for 2 or 3 days postoperatively. Different types and doses of analgesics were applied by anesthetists according to the different conditions of the patients. The Foley catheter was normally retained for at least 5 days. The entire gland was examined and the

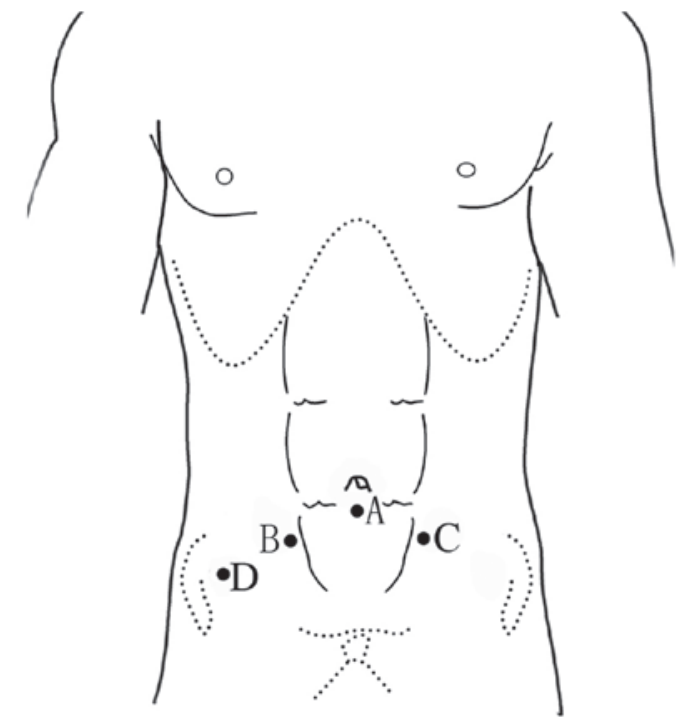

Figure 1. (A) Point at $0.5-1.0 \mathrm{~cm}$ below the umbilicus. (B) Point laterally to the rectus muscle approximately 2 fingers breadth below the umbilicus on the right side. (C) Point laterally to the rectus muscle approximately 2 fingers breadth below the umbilicus on the left side. (D) Point approximately 2 fingers breadth inside the right anterior superior iliac spine.

positive surgical margin (PSM) was defined as any cancer cells in contact with ink. The specimens were examined by an experienced pathologist. Pathological review of the specimens was performed according to the TNM 2002 classification for PCa. Candidates for adjuvant hormonal therapy (AHT) were patients with PSMs, metastasis in the regional lymph node, pathological T3 or T2 disease with Gleason scores $>7$ and initial PSA $>20 \mathrm{ng} / \mathrm{ml}$. AHT comprised combined androgen blockade with luteinizing hormone-releasing hormone analogue (goserelin acetate) injections on a monthly basis and antiandrogen (bicalutamide, $50 \mathrm{mg}$ ) on a daily basis. Candidates for adjuvant external beam radiotherapy (EBRT) were patients with PSMs, seminal vesicle invasion, pathological T3 or T4 disease and postoperative continuous rising PSA values. The patients required digital rectal examination (DRE) and PSA detection postoperatively every 3 months for 2 years, then every 6 months for 5 years and then annually.

Continence and erectile function were evaluated pre- and postoperatively in all patients. Continence was defined when patients did not require incontinence pads. Potency was defined by positive answers to the following questions: 'Could you get and keep an erection?', 'how often were your erections hard enough to be inserted into your partner's vagina?', 'how often were you able to maintain your erection after you had penetrated your partner during sexual intercourse?', 'did your erections last long enough to have sexual intercourse?' and 'were you satisfied with this sexual experience?'. Patients were contacted by telephone and answers to the above questions were recorded individually. Questionnaires were mailed to patients without a telephone interview.

Statistical analysis. Perioperative data were collected, while continence and erectile function results were recorded during the follow-up. Groups were compared using the Pearson Chi-square $\left(\chi^{2}\right)$ analysis. Multivariate analysis of the Cox 
Table I. Perioperative data from present and previous studies.

\begin{tabular}{|c|c|c|c|c|c|}
\hline & Present data & Rozet et al (9) & Stolzenburg et al (10) & Eden et al (11) & Do et al (12) \\
\hline Number of patients & 329 & 600 & 2400 & 1000 & 100 \\
\hline Age (years) (median) & $67(45-78)$ & $62(47-73)$ & $63.3(41-81)$ & $62.0(40-78)$ & $63.7(43-78)$ \\
\hline $\begin{array}{l}\text { Initial PSA }(\mathrm{ng} / \mathrm{ml}) \\
\text { (median or mean) }\end{array}$ & $17.35(4.58-201.85)$ & $7.4 \pm 6.2$ & $9.8(0.08-93)$ & $7.0(1-50)$ & $9.6(1.5-59.2)$ \\
\hline Biopsy Gleason score (median) & $7(4-10)$ & - & - & - & - \\
\hline $\begin{array}{l}\text { Time for anastomosis } \\
\text { (min) (median) }\end{array}$ & $13(10-28)$ & - & - & - & - \\
\hline $\begin{array}{l}\text { Surgery time }(\min ) \\
\text { (median or mean) }\end{array}$ & $90(80-185)$ & $173 \pm 85(95-300)$ & $150.7(50-340)$ & $177(78-600)$ & $150(75-270)$ \\
\hline $\begin{array}{l}\text { Estimated blood loss }(\mathrm{ml}) \\
\text { (median or mean) }\end{array}$ & $75(20-800)$ & $380 \pm 210(20-3500)$ & $255(20-1200)$ & $200(10-1300)$ & $245(30-800)$ \\
\hline Blood transfusion (ml) (median) & $600(200-800)$ & - & - & - & - \\
\hline $\begin{array}{l}\text { Placement time of retropubic drain } \\
\text { (days) (median) }\end{array}$ & $3(2-10)$ & - & - & - & - \\
\hline $\begin{array}{l}\text { Postoperative catheterization time } \\
\text { (days) (median) }\end{array}$ & $6(5-14)$ & $7.6(3-20)$ & $6.19(3-40)$ & $10.0(0.8-120)$ & $6.7(4-24)$ \\
\hline Time to oral intake (days) (median) & $2(1-4)$ & - & - & - & - \\
\hline Time to ambulation (days) (median) & $2(2-4)$ & - & - & - & - \\
\hline $\begin{array}{l}\text { Postoperative hospital stay } \\
\text { (days) (median) }\end{array}$ & $7(6-16)$ & $6.3(4-14)$ & - & $3.0(2-28)$ & - \\
\hline \multicolumn{6}{|l|}{ Pathological TNM } \\
\hline pT2 (Organ confined) & $211(64.2 \%)$ & $432(72 \%)$ & $1693(70.5 \%)$ & - & - \\
\hline pT3a (Extracapsular extension) & $51(15.5 \%)$ & $115(19.2 \%)$ & $473(19.7 \%)$ & - & - \\
\hline pT3b (Seminal vesicle invasion) & $60(18.2 \%)$ & $53(8.8 \%)$ & $226(9.4 \%)$ & - & - \\
\hline $\begin{array}{l}\text { pT4 (Invasion of rectum, } \\
\text { levator muscles and/or pelvic wall) }\end{array}$ & $7(2.1 \%)$ & - & $8(0.3 \%)$ & - & - \\
\hline
\end{tabular}

PSA, prostate-specific antigen.

proportional hazard model was used to identify whether variables had significant independent correlations with biochemical recurrence-free survival. The Kaplan-Meier method and log-rank test were used to compare the biochemical recurrence-free survival among the different groups. In all analyses, $\mathrm{P}<0.05$ was considered to indicate a statistically signifcant difference, and was two-sided. The data were analyzed using SPSS 18.0 software.

\section{Results}

Perioperative data. No conversion or re-intervention were found in the 329 cases of ELRP. Surgery time was recorded from the beginning of subumbilical incision and ended when the specimen was removed. The operative data are shown in Table I, including the median times for anastomosis, surgery, postoperative catheterization and the median estimated blood loss and median blood transfusion.

There were 12 temporary urinary leakages requiring prolonged catheterization to 14 days. A total of 1 case of deep vein thrombosis occurred and was cured by non-surgical
Table II. Percentage of initial PSA, clinical stage and biopsy Gleason score.

No. $(\%)$

$\begin{array}{lc}\text { Initial PSA }(\mathrm{ng} / \mathrm{ml}) & \\ <10 & 108(32.8) \\ 10-20 & 98(29.8) \\ >20 & 123(37.4) \\ \text { Clinical stage } & \\ \text { T1b } & 30(9.1) \\ \text { T1c } & 38(11.5) \\ \text { T2a } & 136(41.3) \\ \text { T2b } & 28(8.6) \\ \text { T2c } & 97(29.5) \\ \text { Biopsy Gleason score } & \\ 2-6 & 135(41.1) \\ 7 & 138(41.9) \\ 8-10 & 56(17.0)\end{array}$

PSA, prostate-specific antigen. 
Table III. PSM and pathological Gleason scores among the differerent NHT groups.

\begin{tabular}{|c|c|c|c|c|c|c|}
\hline & No NHT & NHT $<1$ month & NHT 1-3 months & NHT $>3$ months & Total & P-value \\
\hline \multicolumn{7}{|l|}{$\begin{array}{l}\text { Pathological Gleason } \\
\text { scores }\end{array}$} \\
\hline No Gleason score & 0 & 12 & 5 & 21 & 38 & $\mathrm{P}<0.001$ \\
\hline $2-6$ & 53 & 10 & 0 & 18 & 81 & \\
\hline 7 & 65 & 11 & 13 & 23 & 112 & \\
\hline $8-10$ & 40 & 23 & 20 & 15 & 98 & \\
\hline Total & 158 & 56 & 38 & 77 & 329 & \\
\hline \multicolumn{7}{|l|}{ Surgical margin } \\
\hline PSM & 36 & 8 & 5 & 6 & 55 & $\mathrm{P}=0.027$ \\
\hline NSM & 122 & 48 & 33 & 71 & 274 & \\
\hline Total & 158 & 56 & 38 & 77 & 329 & \\
\hline
\end{tabular}

PSM, positive surgical margin; NHT, neoadjuvant hormonal therapy; NSM, negative surgical margin. P-values were obtained using the Pearson $\chi^{2}$ test: Gleason score of 74.101 and a surgical margin of 9.167 .

Table IV. PSM among different pathological stages and Gleason grade groups.

\begin{tabular}{lrrrr}
\hline & PSM & NSM & Total & P-value \\
\hline Pathological stage & & & & \\
pT2a & 0 & 90 & 90 & \\
pT2b & 0 & 28 & 28 & \\
pT2c & 10 & 83 & 93 & P<0.001 \\
pT3a & 22 & 29 & 51 & \\
pT3b & 16 & 44 & 60 & \\
pT4 & 7 & 0 & 7 & \\
Pathological Gleason & & & & \\
score & & & & \\
No Gleason score & 2 & 36 & 38 & \\
2-6 & 3 & 76 & 79 & \\
7 & 20 & 94 & 114 & P<0.001 \\
8-10 & 30 & 68 & 98 & \\
\hline
\end{tabular}

PSM, positive surgical margin; NSM, negative surgical margin. P-values were obtained using the Pearson $\chi^{2}$ test: pathological stage of 90.770 and Gleason score of 26.698 .

treatment 3 months postoperatively. There was 1 case of alimentary tract hemorrhage which was controlled by the usage of antiulcerative drugs. A total of 7 cases of anterior urethral stricture were identified 3 months postoperatively, which were successfully treated by endoscopic urethrotomy. No rectal injury, lymphocele, incision infection or incision hernia were identified in our patients.

PSM and Gleason score. The median pathological Gleason score was 7 (range, 5-10). The pathological Gleason scores could not be identified in 38 cases that had received preoperative NHT. The number of PSMs in the no NHT group, NHT $<1$ month, NHT 1-3 months and NHT $>3$ months are shown in Table III. The PSM rate was higher in the group without preoperative NHT. Preoperative NHT had a significant effect on pathological Gleason score $(\mathrm{P}<0.001)$ and surgical margin $(\mathrm{P}=0.027)$, respectively. $\mathrm{PSMs}$ were identified in 55 patients $(16.7 \%)$ (Table IV). Pearson Chi-square $\left(\chi^{2}\right)$ analysis demonstrated that PSM correlated with pathological stage and Gleason score, respectively (both $\mathrm{P}<0.001$ ).

A total of 149 patients $(45 \%)$ underwent bilateral standard PLND. Unilateral metastases in the regional lymph nodes were identified in $5 \mathrm{~T} 3 \mathrm{a}$ and $3 \mathrm{~T} 3 \mathrm{~b}$ patients. Bilateral metastases in the regional lymph nodes were identified in $3 \mathrm{~T} 3 \mathrm{~b}$ patients. Postoperatively, 125 (40.0\%) patients had undergone AHT and $35(11.2 \%)$ patients had received both AHT and adjuvant radiotherapy.

A total of 17 patients were lost during the follow-up period. Slight stress urinary incontinence was identified in almost every patient after the catheter was removed. In a minimum follow-up of 12 months (range, 12-72), 216 and 74 patients were completely continent after 3 months and 4-12 months, respectively. A total of 22 patients used 1 precautionary pad daily after 12 months postoperatively and achieved full continence several months later. Neither postoperative persistent urinary leak nor anastomotic stricture had occurred. A total of 139 patients had undergone bilateral NVB sparing procedures and 132 were followed-up. Overall, 29 patients were satisfied with their erection, hardness and duration 12 months postoperatively. During the same postoperative period of time, 17 patients had an erection with inadequate hardness and duration. Younger patients ( $\leq 67$ years of age) had a more rapid continence recovery and a better postoperative potency (Table V).

During the follow-up period of 12-72 months, the patients survived and $89(28.6 \%)$ patients were diagnosed with biochemical recurrence for 2 consecutive PSA values $\geq 0.2 \mathrm{ng} / \mathrm{ml}$. The overall biochemical recurrence-free survival decreased during the follow-up period. Multivariate analyses using the Cox proportional hazard model identified the initial PSA value, PSM, pathological stage and Gleason score as independent prognostic factors for biochemical recurrence-free survival (Fig. 2A-E). 

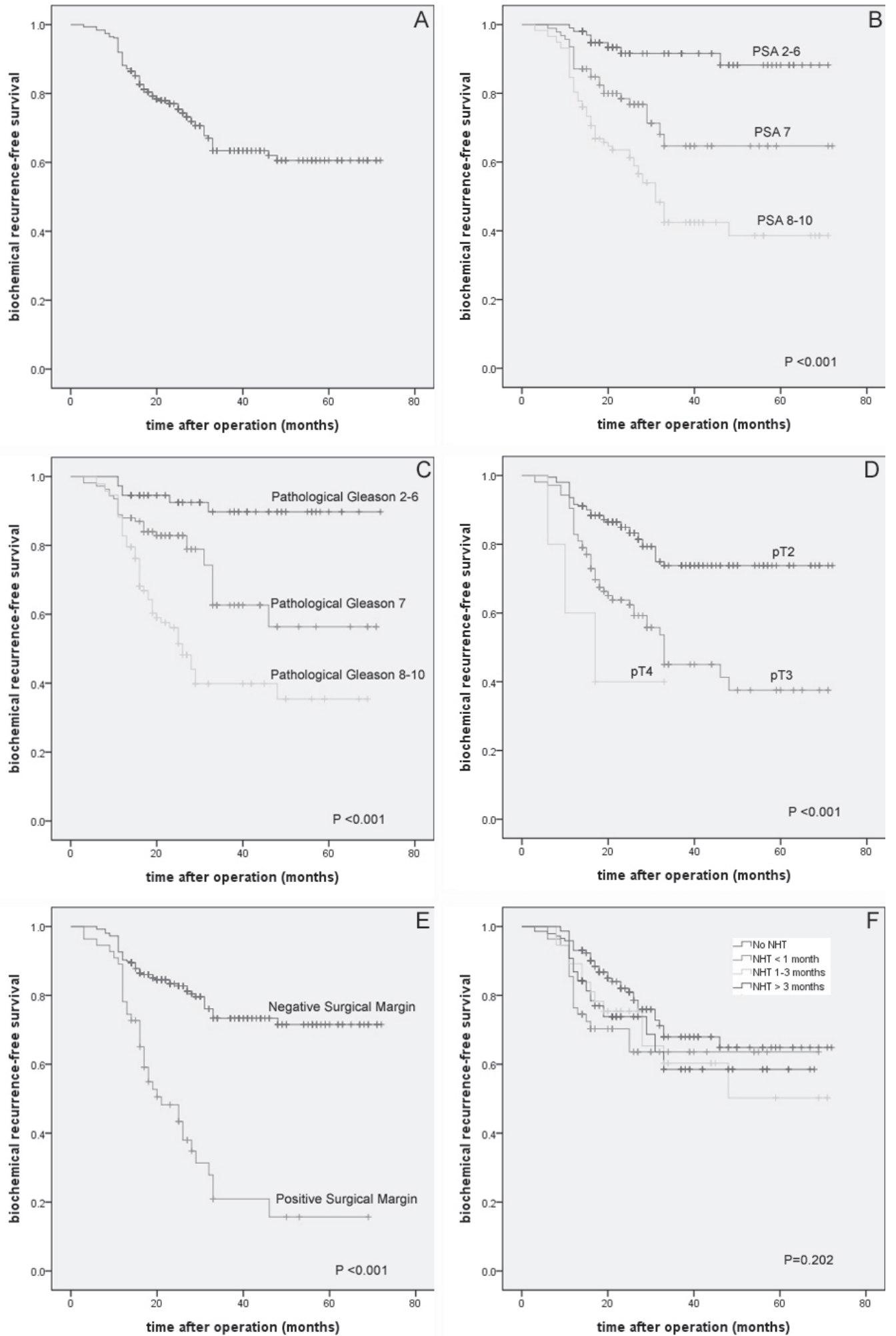

Figure 2. (A) The overall biochemical recurrence-free survival. Biochemical recurrence-free survival according to (B) initial PSA value (P<0.001, log-rank test), (C) Gleason score $(\mathrm{P}<0.001$, log-rank test), (D) pathological stage $(\mathrm{P}<0.001, \log$-rank test), (E) surgical margin $(\mathrm{P}<0.001, \log$-rank test) and (F) preoperative NHT $(\mathrm{P}=0.202, \log$-rank test). PSA, prostate-specific antigen; NHT, neoadjuvant hormonal therapy.

\section{Discussion}

In 1997, the first studies of transperitoneal laparoscopic radical prostatectomy (TLRP) and the initial experience of ELRP were reported by Schuessler et al (6) and Raboy et al (7), respectively. In 2002, Stolzenburg et al (5) introduced their technique and experience of the endoscopic extraperitoneal radical prostatectomy (EERPE). These authors reported that extraperitoneal endoscopic access was able to prevent intraabdominal complications, including bowel injury, ileus or 
Table V. Return of continence and potency are age-related and better in younger males.

\begin{tabular}{|c|c|c|c|c|}
\hline & Age $\leq 67$ years & Age $>67$ years & Total & P-value \\
\hline Complete continence & & & & $\mathrm{P}<0.001$ \\
\hline 3 months postoperatively & 120 & 96 & 216 & \\
\hline 3-12 months postoperatively & 22 & 52 & 74 & \\
\hline Total & 142 & 148 & 290 & \\
\hline Potency at 12 months postoperatively & & & & $\mathrm{P}<0.001$ \\
\hline Erectile dysfunction & 33 & 53 & 86 & \\
\hline Erection with inadequate hardness and duration & 13 & 4 & 17 & \\
\hline Satisfied erection, hardness and duration & 22 & 7 & 29 & \\
\hline Total & 68 & 64 & 132 & \\
\hline
\end{tabular}

P-values were obtained using the Pearson $\chi^{2}$ test: complete continence of 14.711 and potency of 17.069.

intestinal adhesions, and combined the advantages of minimally invasive laparoscopy and retropubic open prostatectomy.

Our initial 20 cases of laparoscopic radical prostatectomy were performed using the transperitoneal approach in 2003. Although neither bowel injury nor ileus occurred, the interference of the bowel and prevention of intraperitoneal complications resulted in the extraperitoneal approach being selected. There were enough cases of transperitoneal approach to compare with the extraperitoneal approach. Our 329 cases of successfully performed ELRP proves that the extraperitoneal approach is a feasible and reproducible technique.

The initial 23 cases of running urethrovesical anastomosis were performed using the single knot method (SKM) (8). We found that the two suture lines were easily interacted in the narrow Chinese pelvic section. When the transperitoneal approach was adopted, intestinal canals made the condition increasingly difficult. We adopted the single needle method (SNM) (4) to successfully perform the running urethrovesical anastomosis. We used a UR-6 taper needle with 5/8 radian; this suitable needle was small enough, allowing for easier rotation in the narrow pelvis. Due to the low friction characteristic of the Monocryl, the sutures glided smoothly under traction, and the poliglecaprone was strong enough when the urethral stump and the bladder neck were brought together with traction. Until the 8 o'clock position of the posterior was sutured, we had not pulled the bladder neck with the urethra immediately after every suture. The tension of anastomosis was shared by several sutures, therefore dilaceration of the bladder or the urethra was avoided. Correct tension of traction on each suture followed with the locking suture at 9 o'clock was a key point.

The majority of the temporary urinary leakages were identified in the initial cases. Upon increasing experience, temporary urinary leakage seldom occurred. The details of SNM and initial results compared with the results of several other methods of urethrovesical anastomosis that have been published (4). A short median time for anastomosis, a short median placement time of the retropubic drain, no postoperative persistent urinary leakage and no anastomotic stricture indicated that SNM was a simple and efficient method.

Rozet et al (9), Stolzenburg et al (10), Eden et al (11) and Do et al (12) have reported their encouraging experiences and results of laparoscopic radical prostatectomy. The majority of our perioperative results were analogical with data from the European studies (Table I). In China, PSA monitoring has not been regularly inspected in older males, thus a quantity of Chinese PCas were identified with high PSA values and late-stages. The median PSA of 17 demonstrated in our study is a little higher than that in a number of other published studies, we consider that this may be due to the fact that there are higher proportions of pT3b and pT4 cases in our patients.

The median age of our patients was 67 years old, thus patients were divided into two groups at the age of 67. Our follow-up data demonstrated that return of continence and potency are age-related and yield better results in younger males ( $\leq 67$ years of age), which is in accordance with other studies by Stolzenburg et al (10), Hu et al (13) and Mendiola et al (14).

A number of Chinese urologists prefer to adopt preoperative NHT in the interval between TURP or biopsy and radical prostatectomy. In our study, over half of the patients had received preoperative NHT for less or more than 3 months. Statistical analysis demonstrated that preoperative NHT had a significant effect on the pathological Gleason score and surgical margin, but not on biochemical recurrence. This result is in accordance with the 2011 EAU guidelines on $\mathrm{PCa}$, which indicates that preoperative NHT prior to radical prostatectomy does not provide a significant overall survival advantage or a significant advantage in disease-free survival over prostatectomy alone. However, NHT substantially improves local pathological variables, including pathological down-staging and PSMs.

Multivariate analyses using the Cox proportional hazard model in this study demonstrated that biochemical recurrencefree survival was statistically significantly correlated with the initial PSA value, pathological Gleason score, pathological stage and PSM. No significant correlation was identified between biochemical recurrence-free survival, body mass index, NHT, AHT and adjuvant radiotherapy, respectively. Since bilateral standard PLND was not performed in all patients, we did not analyse whether lymph node metastasis had a significant independent correlation with biochemical recurrence-free survival. A limitation of our study is that the sample size was small, and that the study was a retrospective analysis with a short postoperative follow-up period, which 
allowed for potential selection bias. Large, prospective studies and long-term follow-up are required to evaluate oncological and functional results of Chinese patients.

In their study, Jemal et al (1) reported that the incidence of PCa in Eastern Asia was 8.2 per 100,000 according to global cancer statistics. A recent unpublished study in Beijing revealed that the incidence of PCa may have been severely underestimated in China. A total of 3,359 males with a mean age of $65 \pm 10$ years received DRE and PSA detection. Overall, 87 cases were identified with abnormal DRE and/or PSA values and 61 cases received transrectal ultrasound-guided biopsy. A total of 19 cases of PCa were diagnosed and 11 cases were late-stage. The authors suggested that PSA monitoring should be applied more widely in large- and medium-sized cities of China. Due to the increase in Chinese PCa cases the application of PSA detection may aid in the diagnosis of $\mathrm{PCa}$, thus an increasing number of urologists may employ ELRP in the future.

\section{Acknowledgements}

This study was supported by the 50th General Financial Grant from China Postdoctoral Science Foundation (Grant no. 201150M1535) and the National Natural Science Foundation for Distinguished Young Scholar (no. 30725040), China.

\section{References}

1. Jemal A, Bray F, Center MM, Ferlay J, Ward E and Forman D: Global cancer statistics. CA Cancer J Clin 61: 69-90, 2011.

2. Gu FL: The incidences of benign prostatic hyperplasia and prostatic cancer in China. Zhonghua Wai Ke Za Zhi 31: 323-326, 1993.
3. Gu F: Epidemiological survey of benign prostatic hyperplasia and prostatic cancer in China. Chin Med J (Engl) 113: 299-302, 2000.

4. Zhang X, Ju Z, Wang C, et al: The single needle method for urethrovesical anastomosis with strengthened posterior fixation during laparoscopic radical prostatectomy. J Huazhong Univ Sci Technolog Med Sci 29: 745-749, 2009.

5. Stolzenburg JU, Do M, Pfeiffer H, König F, Aedtner B and Dorschner W: The endoscopic extraperitoneal radical prostatectomy (EERPE): technique and initial experience. World $\mathbf{J}$ Urol 20: 48-55, 2002.

6. Schuessler WW, Schulam PG, Clayman RV and Kavoussi LR: Laparoscopic radical prostatectomy: initial short-term experience. Urology 50: 854-857, 1997.

7. Raboy A, Ferzli G and Albert P: Initial experience with extraperitoneal endoscopic radical retropubic prostatectomy. Urology 50: 849-853, 1997.

8. Van Velthoven RF, Ahlering TE, Peltier A, Skarecky DW and Clayman RV: Technique for laparoscopic running urethrovesical anastomosis: the single knot method. Urology 61: 699-702, 2003

9. Rozet F, Galiano M, Cathelineau X, Barret E, Cathala N and Vallancien G: Extraperitoneal laparoscopic radical prostatectomy: a prospective evaluation of 600 cases. J Urol 174: 908-911, 2005.

10. Stolzenburg JU, Kallidonis P, Minh D, et al: Endoscopic extraperitoneal radical prostatectomy: evolution of the technique and experience with 2400 cases. J Endourol 23: 1467-1472, 2009.

11. Eden CG, Neill MG and Louie-Johnsun MW: The first 1000 cases of laparoscopic radical prostatectomy in the UK: evidence of multiple 'learning curves'. BJU Int 103: 1224-1230, 2009.

12. Do M, Haefner T, Liatsikos E, et al: Endoscopic extraperitoneal radical prostatectomy after previous transurethral resection of prostate: oncologic and functional outcomes of 100 cases. Urology 75: 1348-1352, 2010

13. Hu JC, Elkin EP, Pasta DJ, Lubeck DP, Kattan MW, Carroll PR and Litwin MS: Predicting quality of life after radical prostatectomy: results from CaPSURE. J Urol 171: 703-707, 2004.

14. Mendiola FP, Zorn KC, Mikhail AA, Lin S, Orvieto MA, Zagaja GP and Shalhav AL: Urinary and sexual function outcomes among different age groups after robot-assisted laparoscopic prostatectomy. J Endourol 22: 519-524, 2008. 